\title{
Effects of Chemically Modified Messenger RNA on Protein Expression
}

\begin{abstract}
Bin Li, ${ }^{\dagger, \#}$ Xiao Luo, ${ }^{\dagger, \#}$ and Yizhou Dong ${ }^{*,+, \neq, \S, \|}$
${ }^{\dagger}$ Division of Pharmaceutics \& Pharmaceutical Chemistry, College of Pharmacy, ${ }^{\ddagger}$ Department of Biomedical Engineering, ${ }^{\S}$ The Center for Clinical and Translational Science, and "The Comprehensive Cancer Center, The Ohio State University, Columbus, Ohio 43210, United States
\end{abstract}

\section{Table of Contents}

Materials 


\section{Experimental Section}

Materials. Rabbit reticulocyte lysate kit and Bright-Glo reagent were purchased from Promega Corporation. Eagle's Minimum Essential Medium (EMEM) was purchased from Corning Incorporated. RPMI-1640 Medium and Hep 3B cells were purchased from American Type Culture Collection. RNeasy Mini Kit was from QIAGEN. Dulbecco's Modified Eagle Medium (DMEM), Opti-MEM medium, fetal bovine serum, Lipofectamine 2000, High Capacity cDNA Reverse Transcription Kits and SYBR Green PCR Master Mix were purchased from Life technologies. Antarctic phosphatase was purchased from New England Biolabs.

Synthesis of chemically modified mRNA. Chemically modified mRNAs encoding firefly Luciferase and green fluorescent protein respectively were synthesized using an in vitro transcription from the corresponding plasmid DNA template by TriLink BioTechnologies. Chemical modified nucleotides were completely substituted for their natural counterparts while synthesizing the mRNAs. The transcripts were then optimized for mammalian systems with 5' cap and 3' poly(A) tail structures. mRNAs were purified using commercially available silica-based spin columns. mRNAs were dephosphorylated with Antarctic phosphatase and repurified by silica-based spin column.

mRNA translation in rabbit reticulocyte lysate system. Two microliter of FLuc mRNA $(1 \mu \mathrm{g} / \mu \mathrm{L})$ was added to $48 \mu \mathrm{L}$ of rabbit reticulocyte lysate reaction assembly and the mixture was incubated at either $30{ }^{\circ} \mathrm{C}$ or $37{ }^{\circ} \mathrm{C}$ for $90 \mathrm{~min}$. After incubation, $50 \mu \mathrm{L}$ of the substrate consisting of $25 \mu \mathrm{L}$ of Bright-Glo and $25 \mu \mathrm{L}$ of PBS was added to $2.5 \mu \mathrm{L}$ of the reaction mixture. Luminescence intensity was measured immediately after 5 minutes dark incubation.

mRNA translation in cells. Hep 3B cells were cultured in Eagle's Minimum Essential Medium (EMEM), THP-1 cells in RPMI-1640 Medium and primary rat hepatocytes in Dulbecco's Modified Eagle Medium (DMEM). All medium supplemented with 10\% fetal bovine serum. For FLuc mRNA translation, Hep 3B and THP-1 cells were seeded in 96-well white plates at a density of $10^{4}$ cells/well. Following overnight culture, 50 ng of modified FLuc mRNA was complexed with Lipofectamine 2000 in Opti-MEM medium, and the mixture was added to each well after 5 min incubation. Twenty 
four hours post-transfection, $100 \mu \mathrm{L}$ of Bright-Glo reagent was added to each well, and luminescence intensity was measured immediately after 5 minutes dark incubation. For eGFP mRNA translation, Hep 3B, THP-1 cells and primary rat hepatocytes were seeded in 6-well plates at a density of $2.0 \times$ $105 /$ well. After overnight culture, $2 \mu \mathrm{g}$ of modified FLuc mRNA was complexed with Lipofectamine 2000 in Opti-MEM medium, and the mixture was added to each well after 5 min incubation. After 24 hours treatment, cells were harvested and the green fluorescence intensity was determined on a BD LSR II flow cytometer (BD Biosciences, San Jose, CA).

RNA isolation, cDNA synthesis and quantitative PCR (qPCR). Hep 3B cells were seeded in 6-well plates at a density of $3 \times 10^{5}$ cells/well and cultured overnight. $1 \mu \mathrm{g}$ of eGFP mRNA complexed with Lipofectamine 2000 in Opti-MEM medium was tansfected into cells. After one hour incubation, cells were rinsed twice to remove the residual RNA and incubated with fresh medium. At desired time points $(\mathrm{t}=0$, 1.5, 3 and $4.5 \mathrm{~h})$, total RNAs were extracted with RNeasy Mini Kit, reverse transcribed into complementary DNA (cDNA) by High Capacity cDNA Reverse Transcription Kits, and quantified with gene-specific primers (Table S1) and SYBR Green PCR Master Mix in a StepOne Plus Real Time PCR system (Applied Biosystems, Foster City, CA) according the manufacturer's instruction. The expression of the target gene versus that of the reference gene (18S rRNA) was calculated using formula $2^{-\Delta \Delta \mathrm{Ct}}$, where $\mathrm{C}_{\mathrm{t}}$ was the cycle threshold value. The relative eGFP mRNA levels at different time points were normalized to the initial $(\mathrm{t}=0)$ eGFP mRNAs. 


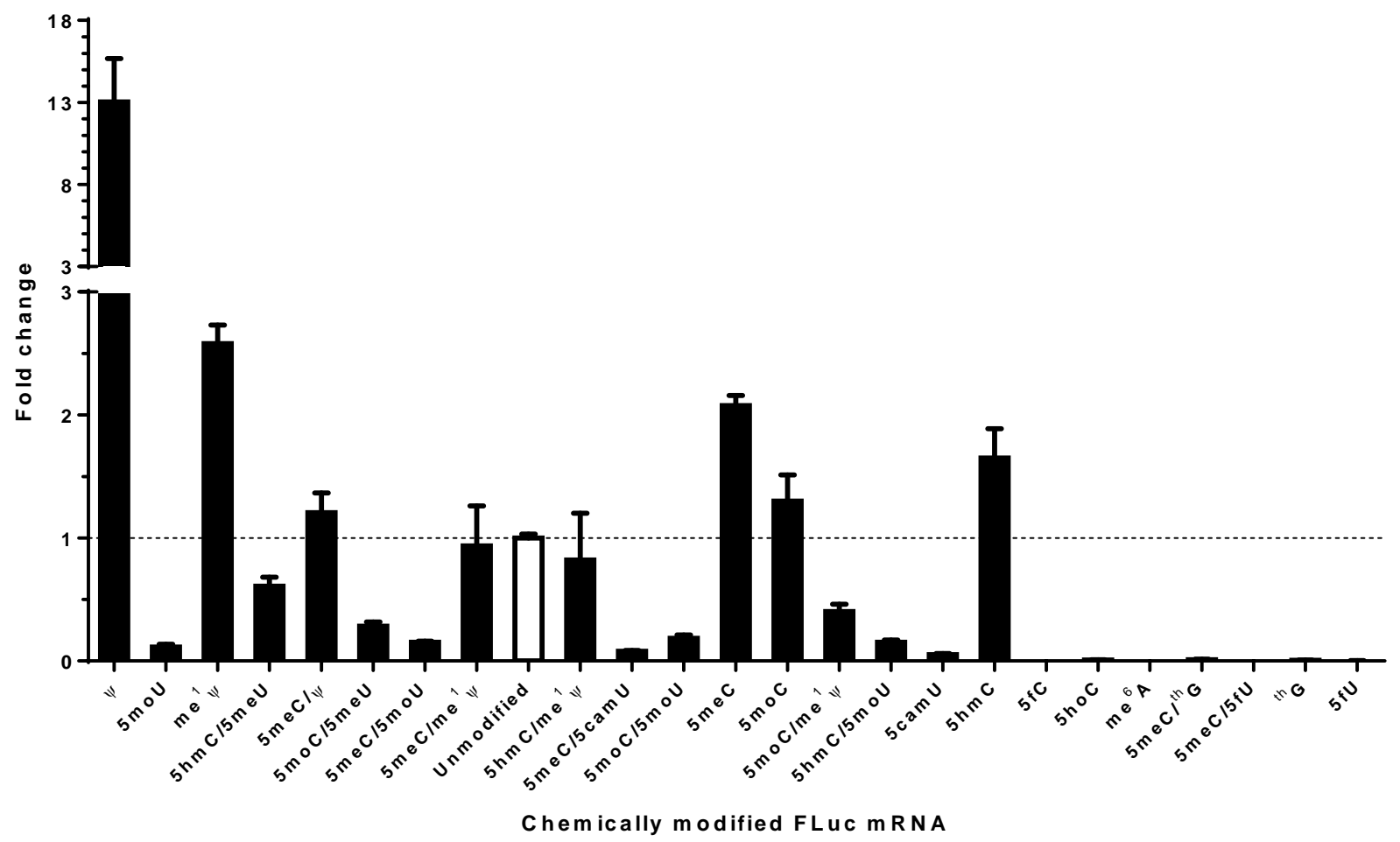

Figure S1. Relative FLuc expression of 24 chemically modified FLuc mRNAs in Hep 3B cells (normalized by unmodified FLuc mRNA).

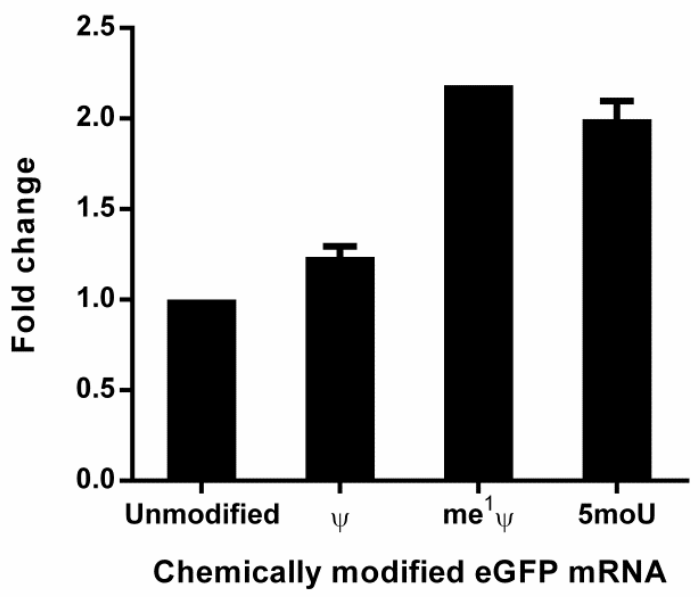

Figure S2. Relative eGFP expression in primary rat hepatocytes normalized by unmodified eGFP mRNA. $\psi$-, me ${ }^{1} \psi$-, and 5 moU-modified eGFP mRNAs were more potent than unmodified eGFP mRNA. 
Table S1. List of gene-specific oligonucleotide primer sets used for SYBR Green real-time qPCR.

\begin{tabular}{lll}
\hline Name & Forward primer sequence & Reverse primer sequence \\
18S rRNA & 5'-GCT CTA GAA TTA CCA CAG TTA TC-3' & 5'-AAA TCA GTT ATG GTT CCT TTG GT-3' \\
eGFP & 5'-ACG TAA ACG GCC ACA AGT TC-3' & 5'-AAG TCG TGC TGC TTC ATG TG-3' \\
\hline
\end{tabular}

